\title{
Examination of Deformation Mechanisms of Magnesium AZ31: in situ X-Ray Diffraction and Self-Consistent Modelling
}

\author{
M. Wronski ${ }^{1, a}$, K. Wierzbanowski ${ }^{1, b^{*}}, A$. Baczmanski ${ }^{1, c}$, S. Wronski ${ }^{1, d}$, \\ M. Wojtaszek ${ }^{1, \mathrm{e}}$, R. Wawszczak ${ }^{1, \mathrm{f}}$ and M. Muzyka ${ }^{1, \mathrm{~g}}$ \\ ${ }^{1} \mathrm{AGH}$ University of Science and Technology, Faculty of Physics and Applied Computer Science, \\ al. Mickiewicza 30, 30-059 Kraków, Poland \\ a mwronskii@gmail.com, bwierzbanowski@fis.agh.edu.pl, ' baczmanski@fis.agh.edu.pl, \\ dwronski.sebastian@gmail.com, ${ }^{\mathrm{e}}$ mich.wojtaszek@gmail.com, \\ fwawszczak@fis.agh.edu.pl, 9maciejmuzyka@gmail.com
}

Keywords: Magnesium AZ31, Slip Systems, X-ray Diffraction, Texture, Self-consistent Model

\begin{abstract}
Samples of rolled commercial AZ31 magnesium were stretched along RD and in situ $\mathrm{X}$-ray measurements were performed. The macroscopic stress-strain curves were examined in order to find basic mechanical material parameters. The lattice strains for different $h k l$ reflections were determined as a function of the applied tensile stress. The obtained experimental results were compared with the predictions of the elasto-plastic self-consistent model. It was found that mainly slip systems are responsible for the observed material deformation and their critical resolved shear stresses (CRSS) were evaluated.

\section{Introduction}

One observes a growing interest in magnesium and its alloys in the last decade (e.g., [1]). Magnesium due to its unique properties, finds many technological application. It has an advantageous ratio of the yield strength and mass density. The disadvantage of this material is a low ductility at room temperature, which severely limits its formability. Therefore, it is necessary to better understand its deformation mechanisms, which can help to optimize its formability. The aim of this work was an examination of the mechanisms of plastic deformation of magnesium AZ31 basing on X-ray studies carried out during in situ tensile tests. The tensile force was applied along the rolling direction of the initial material. In this experiment two kinds of data were obtained: crystal lattice strains for different reflections and the macroscopic stressstrain curve. In order to determine selected material parameters the experimental results were compared with deformation model predictions. The elasto-plastic self-consistent model was used in the present study $[2,3]$. The slip and twinning systems, which were taken into account in the calculations are listed in Tables 1 and 2.

Generally, a comparison of experimental and calculated lattice strains vs. applied load allows the identification of active deformation mechanisms of the examined material, i.e., slip and twinning systems and the estimation of their critical resolved shear stresses (CRSS). Such a study can also provide information on the activity of deformation mechanisms and their influence on the observed material characteristics.
\end{abstract}


Table 1. Slip systems of magnesium [4,5] and values of their critical resolved shear stresses (CRSS), which led to the best agreement between measured and predicted lattice strains.

\begin{tabular}{lcc}
\hline Slip system & Miller indices & CRSS [MPa] \\
\hline Basal B & $\{0001\}\langle 11 \overline{2} 0\rangle$ & 17 \\
Prismatic $\mathrm{P}$ & $\{1 \overline{1} 00\}\langle 11 \overline{2} 0\rangle$ & 75 \\
Pyramidal $\pi<\mathrm{a}>$ & $\{1 \overline{1} 01\}\langle 11 \overline{2} 0\rangle$ & 75 \\
Pyramidal $\pi_{1}<\mathrm{c}+\mathrm{a}>$ & $\{0 \overline{1} 11\}\langle 11 \overline{2} 3\rangle$ & 80 \\
Pyramidal $\pi_{2}<\mathrm{c}+\mathrm{a}>$ & $\{11 \overline{2} \overline{2}\}\langle 11 \overline{2} 3\rangle$ & 70
\end{tabular}

Table 2. Twinning systems of magnesium.

\begin{tabular}{cl}
\hline Twinning systems & Miller indices \\
\hline Compression twins & $\{11 \overline{2} 2\}\langle\overline{1} \overline{1} 23\rangle$ \\
Tensile twins & $\{10 \overline{1} 2\}\langle\overline{1} 011\rangle$
\end{tabular}

\section{Experimental}

The initial samples were cut along the rolling direction (RD) from the commercial AZ31 sheet, with chemical composition shown in Table 3. Its microstructure was examined by the EBSD technique, using the Stereoscan Cambridge S360 microscope. The orientation map of inverse pole figure for the normal direction (ND) is shown in Fig. 1. The average grain size, as determined from EBSD measurements, was around $12 \mu \mathrm{m}$. Texture of the initial material contained a predominating $<0001>\| R D$ fibre component, which is clearly visible on the $\{0001\}$ pole figure - Fig. 2 . This strong texture implies a predominating orientation of hexagonal crystal unit cells shown schematically in Fig. 3.

Table 3. Chemical composition of AZ31.

\begin{tabular}{|l|l|l|l|l|l|}
\hline Element & $\mathrm{Al}$ & $\mathrm{Zn}$ & $\mathrm{Mn}$ & $\mathrm{Cu}$ & $\mathrm{Mg}$ \\
\hline Mass fraction [\%] & $2.5-3.5$ & $0.7-1.3$ & $0.2-1.0$ & 0.05 & $94.15-96.55$ \\
\hline
\end{tabular}
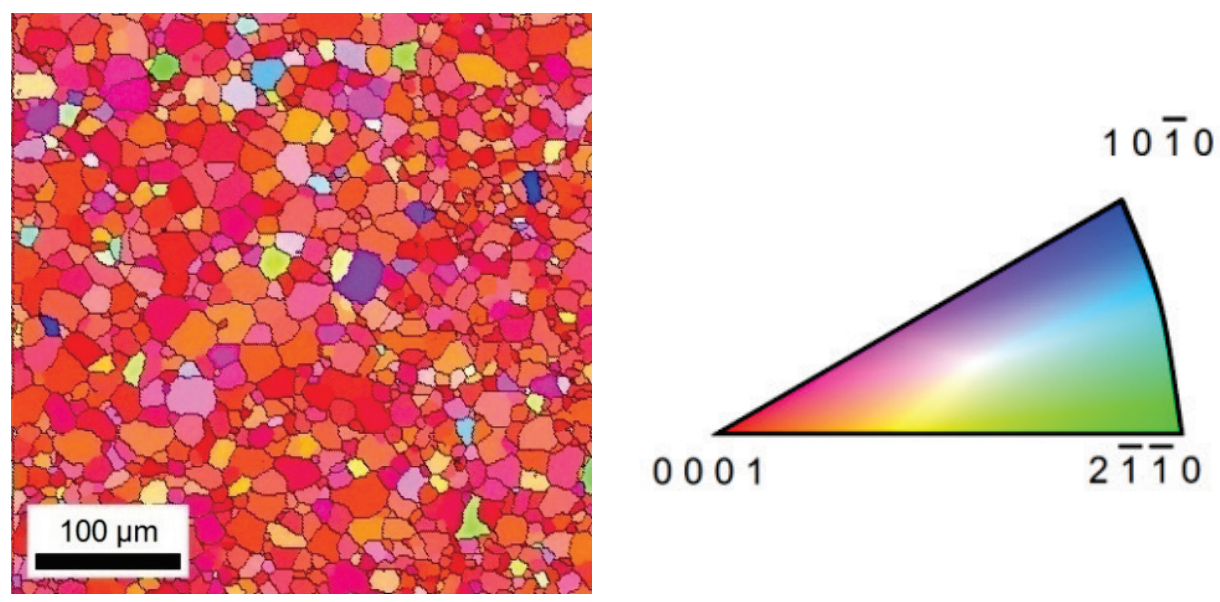

Fig. 1: Initial microstructure of the examined magnesium alloy AZ31. EBSD map of inverse pole figure for the sample normal direction (ND) is shown. 

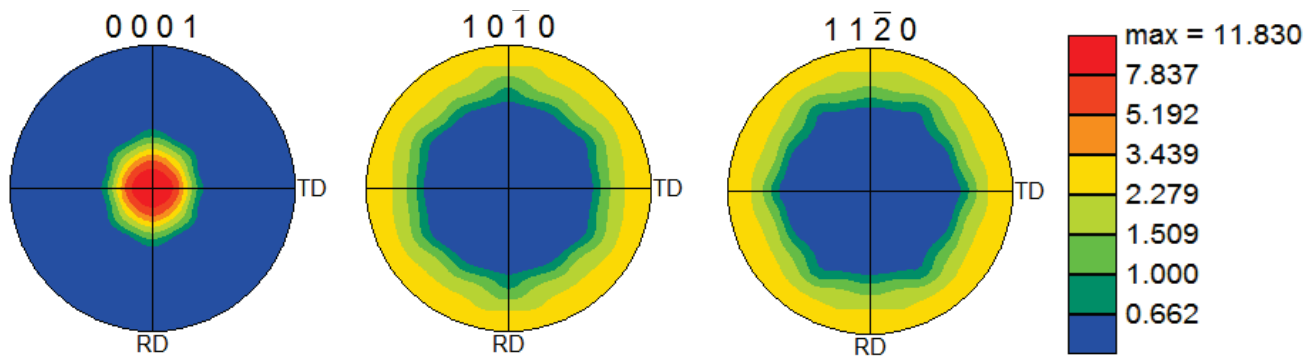

Fig. 2: Determined pole figures for the examined magnesium sample.

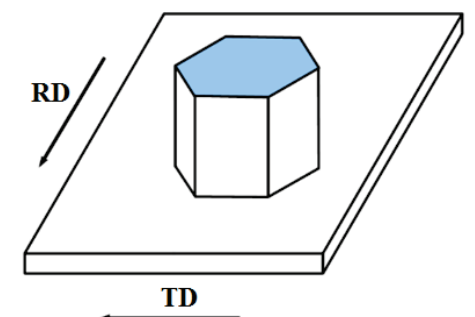

Fig. 3: Predominating position of lattice cells of grains expressed in the sample coordinate system defined by the primary rolling process (RD and TD - rolling and transverse directions).

The majority of crystallites exhibit orientations rotated around c-axis corresponding to an $<0001>$ axial texture.

The scheme of the diffraction experiment is shown in Fig. 4. The X-ray in-situ measurements were done during tensile tests.

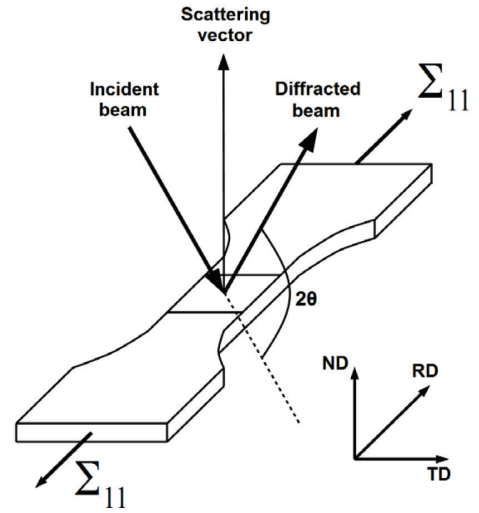

Fig. 4: X-ray diffraction geometry used for in-situ measurements during tensile tests.

\section{Results and discussion}

The measured and predicted macroscopic stress-strain curves are shown in Fig. 5. A perfect agreement is obtained between the experiment and model calculation. The elasto-plastic transition occurs in the range between $130 \mathrm{MPa}$ and $140 \mathrm{MPa}$. 


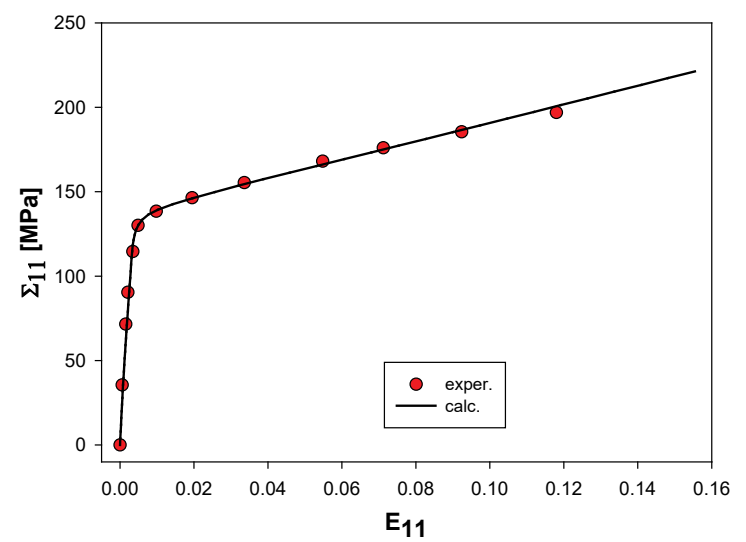

Fig. 5: Comparison between experimental and calculated (self-consistent elasto-plastic model) stress-strain curves.

A more detailed insight into material structure can be done by internal stress measurements (e.g., [6-9]). The diffraction in-situ experiments were performed using X-ray $\mathrm{Cu}$ radiation and the analysed $h k l$ reflections are listed in Table 4.

Table 4. \{hkl\} reflection used to examine the AZ31 sample.

\begin{tabular}{llllllllllll}
\hline $\mathbf{h}$ & 0 & 1 & 1 & 1 & 0 & 1 & 1 & 1 & 0 & 2 & 1 \\
$\mathbf{k}$ & 0 & 0 & 0 & 0 & 0 & 0 & 1 & 0 & 0 & 0 & 0 \\
$\mathbf{l}$ & 2 & 1 & 2 & 3 & 4 & 4 & 4 & 5 & 6 & 5 & 6 \\
\hline
\end{tabular}

The lattice strains were measured along the normal sample direction (ND), i.e., perpendicular to the applied force. The determined lattice strains $\left\langle\varepsilon_{33}\right\rangle_{\{h k l\}}$ versus applied stress component $\Sigma_{11}$, for different $h k l$ reflections, are shown in Fig. 6 . The best fits of the model results to experimental curves are superimposed in these figures. The presented theoretical results were obtained with optimal values of the critical resolved shear stresses (CRSS) listed in Table 1. It is interesting to note that similar values of CRSS were found in the papers $[10,11]$, where neutron diffraction results (i.e., for bulk material) were modelled by the visco-plastic self-consistent model with Voce law for slip systems hardening. Also the results of the present authors, obtained using neutron diffraction and the elasto-plastic deformation model are similar.

It can be noted that for reflections $\{102\},\{103\},\{114\},\{205\}$ some non-linearity is observed in the range of $\Sigma_{11}$ values up to $140 \mathrm{MPa}$ (in principle this is elastic range - according to macroscopic stress-strain curve). This behaviour can be explained by an early activations of selected slip systems (e.g., of basal systems), caused by the presence of residual stresses originating from the preceding rolling process. In the future work this effect should be taken into account and checked by a prior modelling.

In other groups of grains, such as by $\{101\},\{002\},\{106\},\{104\},\{004\},\{105\},\{006\}$ reflections, regular linear relations appear in the elastic range. At $\Sigma_{11}=140 \mathrm{MPa}$ one observes inflection points, which confirms the transition to the plastic range (activation of consecutive slip systems). It can be generally concluded that in all the cases the trends of experimental curves are approximately reproduced by the model predictions. 

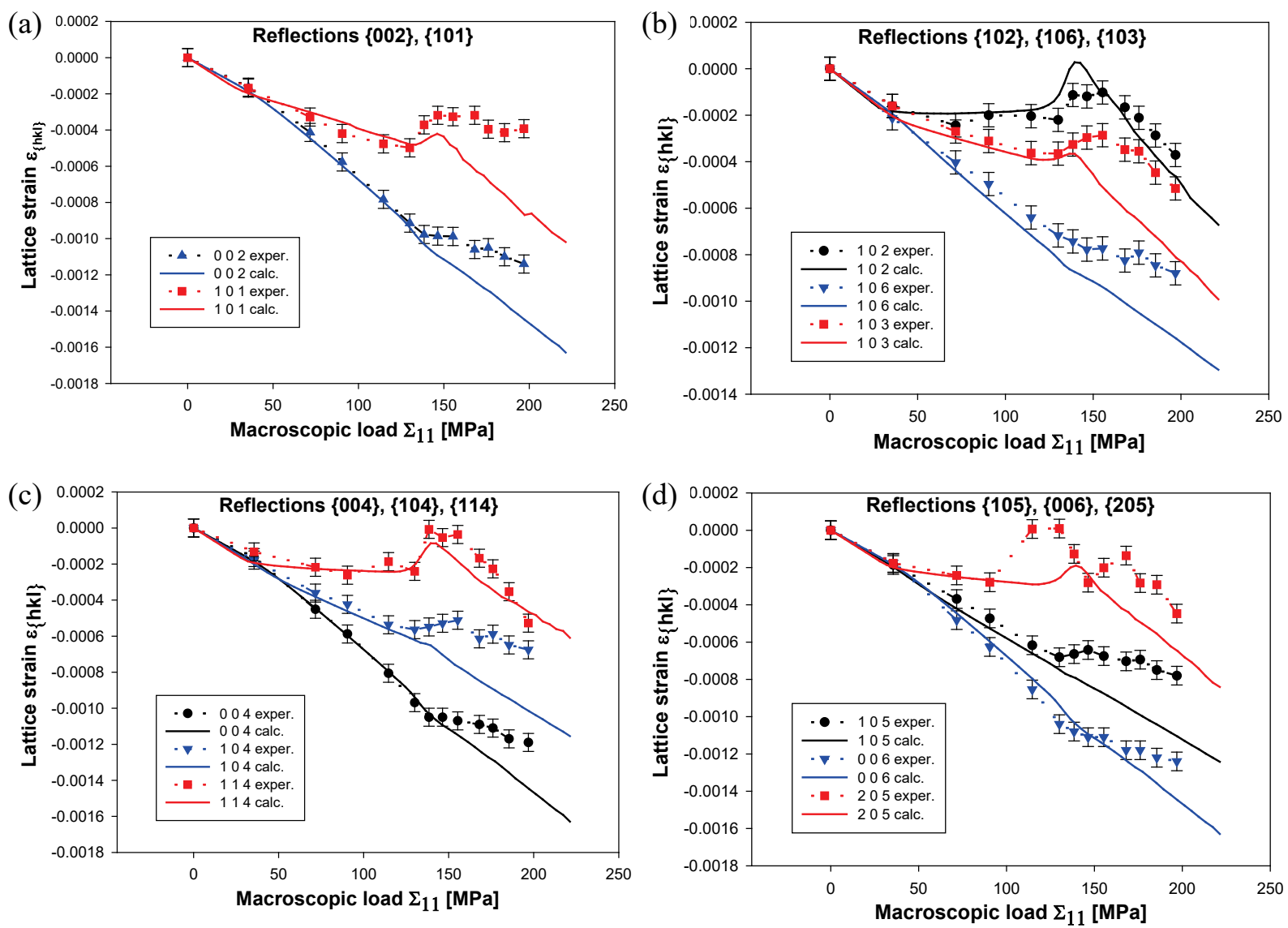

Fig. 6: Lattice strains $\left(<_{33}>\{h k l\}\right)$ in the direction perpendicular to the applied force (i.e., along

ND) versus applied stress component $\sum_{11}$ : experimental results (broken lines with points) are compared with model predictions (solid lines). The results for the following reflections are shown: (a) $\{002\},\{101\}$; (b) $\{102\},\{106\},\{103\}$; (c) $\{004\},\{104\},\{114\}$; (d) $\{105\},\{006\}$, $\{205\}$.

The influence of twinning on the predicted lattice strains was also examined in the frame of a deformation model. The estimated CRSS for the tensile and compressive twinning systems are $50 \mathrm{MPa}$ and $60 \mathrm{MPa}$, respectively(CRSS for tensile twins found in [10,11] is at similar level).

Introduction of this mechanism improves only slightly the agreement between experimental and model results in the case of basic reflection $\{002\}$ (and also the higher order reflections $\{004\}$ and $\{006\}$ corresponding to the same crystallographic plane). In the case of other reflections, e.g., $\{102\}$, it led to worse agreement. Consequently, taking into account only slip systems was sufficient to find the best fit of experimental lattice strains to the calculated ones.

It should be mentioned that the experimentally determined twin volume in magnesium deformed by tension varies between 0 and $4 \%$ for average grain size in the range of 10-20 $\mu \mathrm{m}$ [12]. It is a reason why inclusion of twinning in the calculations did not improve the agreement between experimental and predicted lattice strains.

\section{Conclusions}

The present study of deformation mechanisms of magnesium AZ31 was based on the comparison of measured lattice strains with those predicted by the elasto-plastic self-consistent deformation model. The optimal CRSS values, leading to the best agreement between experimental and theoretical results, were evaluated. Moreover, it was found that slip systems 
play a predominant role in the plastic deformation of magnesium. Inclusion of tensile and compression twinning systems in the calculations did not generally improve the agreement between predicted and experimental results.

\section{Acknowledgments}

This study was financed by grants of the Polish National Centre for Science (NCN) No: DEC2015/19/D/ST8/00818 and DEC-2013/11/B/ST3/03787 and by the AGH statutory works No. 11.11.220.01.

\section{References}

[1] M. Easton, A. Beer, M. Barnett, C. Davies, G. Dunlop, Y. Durandet, S. Blacket, T. Hilditch and P. Beggs, Magnesium alloy applications in automotive structures, JOM 60 (2008) 57-62. https://doi.org/10.1007/s11837-008-0150-8

[2] K. Wierzbanowski, A. Baczmanski, P. Lipinski and A. Lodini, Elasto-plastic models of polycrystalline material deformation and their applications, Arch. Metall. Mater. 52 (2007) 77-86

[3] A. Baczmański, N. Hfaiedh, M. François and K. Wierzbanowski, Plastic Incompatibility Stresses and Stored Elastic Energy in Plastically Deformed Copper, Mat. Sci. Eng. A. 501 (2009) 153-165. https://doi.org/10.1016/j.msea.2008.09.072

[4] Y.N. Wang and J.C. Huang, Texture analysis in hexagonal materials, Mater. Chem. Phys. 81 (2003) 11-26. https://doi.org/10.1016/S0254-0584(03)00168-8

[5] M.J. Philippe, M. Serghat, P. Van Houtte and C. Esling, Modelling of texture evolution for materials of hexagonal symmetry-II. application to zirconium and titanium $\alpha$ or near $\alpha$ alloy, Acta Metall. Mater. 43 (1995) 1619- 1630. https://doi.org/10.1016/0956-7151(94)00329-G

[6] S. Wronski, M. Wrobel, A. Baczmanski and K. Wierzbanowski, Effects of cross-rolling on residual stress, texture and plastic anisotropy in f.c.c. and b.c.c. metals, Mater. Charact. 77 (2013) 116-126. https://doi.org/10.1016/j.matchar.2013.01.005

[7] K. Wierzbanowski, J. Tarasiuk, B. Bacroix, K. Sztwiertnia and P. Gerber, Recrystallization Textures - Two Types of Modelling, Met. Mater. Int. 9 (2003) 9-14. https://doi.org/10.1007/BF03027223

[8] S. Wronski, K. Wierzbanowski, B. Bacroix, M. Wróbel, E. Rauch, F. Montheillet and M. Wroński, Texture heterogeneity of asymmetrically rolled low carbon steel, Arch. Metall. Mater. 54 (2009) 89-102.

[9] M. Marciszko, A. Baczmanski, K. Wierzbanowski, M. Wróbel, C. Braham, J.-P. Chopart, A. Lodini, J. Bonarski, L. Tarkowski and N. Zazi, Application of multireflection grazing incidence method for stress measurements in polished Al-Mg alloy and CrN coating, Appl. Surf. Sci. 266 (2013) 256-267. https://doi.org/10.1016/j.apsusc.2012.12.005

[10] B. Clausen, C.N. Tome, D.W. Brown and S.R. Agnew, Reorientation and stress relaxation due to twinning: Modeling and experimental characterization for Mg, Acta Mater. 56 (2008) 2456-2468. https://doi.org/10.1016/j.actamat.2008.01.057

[11] S.R. Agnew, C.N. Tome, D.W. Brown, T.M. Holden and S.C. Vogel, Study of slip mechanisms in a magnesium alloy by neutron diffraction and modeling, Scripta Mater. 48 (2003) 1003-1008. https://doi.org/10.1016/S1359-6462(02)00591-2

[12] A. Jain, O. Duygulu, D.W. Brown, C.N. Tome and S.R. Agnew, Grain size effects on the tensile properties and deformation mechanisms of a magnesium alloy, AZ31B, sheet, Mat. Sci. Eng. A. 486 (2008) 545-555. https://doi.org/10.1016/j.msea.2007.09.069 